\title{
PROHEXADIONA DE CÁLCIO E A REDUÇÃO DO VIGOR, PRODUTIVIDADE E QUALIDADE DA UVA GRANO D'ORO ${ }^{1}$
}

\author{
LARISSA VILLAR ${ }^{2}$, ALBERTO FONTANELLA BRIGHENTI ${ }^{3}$, \\ RICARDO CIPRIANI ${ }^{4}$, APARECIDO LIMA DA SILVA ${ }^{5}$
}

RESUMO - A Prohexadiona de Cálcio é um regulador de crescimento que atua na inibição da biossíntese de giberelinas, reduzindo o crescimento vegetativo. O objetivo deste trabalho foi avaliar seu efeito na videira Grano d'Oro, mutação da variedade Bordô (Vitis labrusca L.), na redução do vigor, produtividade e qualidade dos frutos. Foi utilizado o produto Apogee ${ }^{\circledR}$, na concentração de $250 \mathrm{mg} \mathrm{L}^{-1}$ de Prohexadiona de cálcio, em diferentes datas de aplicação. Os tratamentos foram uma aplicação na plena florada, uma aplicação 45 dias após a plena florada e testemunha (sem nenhuma aplicação). O delineamento experimental foi em blocos completamente casualizados, com 4 blocos e 20 plantas por parcela. Ramos marcados foram medidos para a determinação do seu crescimento, e na colheita foi determinado o número de cachos por planta, a produtividade ( $\mathrm{kg}_{\text {planta }}{ }^{-1}$ e $\mathrm{T} \mathrm{ha}^{-1}$ ), o peso de 50 pagas, o número de bagas por cacho, o teor de sólidos solúveis totais $\left({ }^{\circ} \mathrm{Brix}\right)$, a acidez total titulável $\left(\mathrm{meq} \mathrm{L} \mathrm{L}^{-1}\right)$ e o $\mathrm{pH}$. A aplicação de Prohexadiona de Cálcio na plena florada reduziu o crescimento dos ramos em 33\%, aos 15 dias após a plena florada, diminuindo essa redução ao longo do ciclo, sendo que, aos 93 dias após a plena florada, chegou a $8 \%$ em relação aos outros dois tratamentos. A aplicação da Prohexadiona de Cálcio na plena florada causou as maiores reduções no vigor e o maior aumento na produtividade das plantas de Grano d'Oro. $\mathrm{O}$ aumento da produtividade e a redução do vigor foram acompanhados por uma redução nos teores de sólidos solúveis totais. A redução no vigor não eliminou a necessidade da realização do desponte.

Termos para Indexação: Vitis labrusca L.; crescimento vegetativo, Apoge ${ }^{\circledR}$, regulador de crescimento

\section{PROHEXADIONE-CALCIUM AND VIGOR REDUCTION, PRODUCTIVITY AND QUALITY OF GRANO D'ORO GRAPEVINES}

\begin{abstract}
Prohexadione-calcium is a gibberellin biosynthesis inhibitor used to suppress the vegetative growth. The objective of this work was to evaluate the effect of prohexadione-calcium on vigor reduction, productivity and quality of Grano d'Oro, a mutation of Bordô (Vitis labrusca L.) grapevine. Apogee ${ }^{\circledR}$ was used at a concentration of $250 \mathrm{mg} \mathrm{L}^{-1}$ of Prohexadione-calcium in different spray dates. The treatments were applied at full bloom, 45 days after full bloom and a control (no application). The experimental design was a completely randomized blocks design, with four blocks and 20 plants per plot. Marked branches were measured to assess the growth curve. During harvest, the bunch number per plant, productivity (kg plant ${ }^{-1}$ and $\left.\mathrm{T} \mathrm{ha}^{-1}\right), 50$ berries weight, berry number per cluster, total soluble solids ( ${ }^{\circ}$ Brix), total acidity (meq L $\left.\mathrm{L}^{-1}\right)$ and $\mathrm{pH}$ were determined. Prohexadione-calcium application at full bloom reduced branch growth in 33\% at the $15^{\text {th }}$ day after full bloom, this reduction decreased over the cycle, and at the $93^{\text {th }}$ day after full bloom reached $8 \%$, compared to the other treatments. Prohexadione-calcium application at full bloom caused the higher vigor reduction and the higher productivity increase in Grano d'Oro grapevines. The productivity increase and the vigor reduction ran parallel to a total soluble solids reduction. The vigor reduction did not eliminate the need for shoot topping.
\end{abstract}

Index terms: Vitis labrusca L.; vegetative growth, Apogee ${ }^{\circledR}$, plant growth regulator.

\footnotetext{
${ }^{1}$ Trabalho Sinfruit 195 - Simpósio Internacional de Fruticultura - Avanços na Fruticultura (17 a 21 Outubro)

${ }^{2}$ Engenheira Agrônoma, doutoranda em Recursos Genéticos Vegetais RGV-UFSC; Rodovia Admar Gonzaga, 1346, Itacorubi, Florianópolis -SC, CEP 88040-900. E-mail: larissavillar.agro@gmail.com

${ }^{3}$ Engenheiro Agrônomo, doutorando em Recursos Genéticos Vegetais RGV-UFSC. E-mail: brighenti_07@hotmail.com ${ }^{4}$ Engenheiro Agrônomo, mestrando em Recursos Genéticos Vegetais RGV-UFSC. E-mail: ciprianiricardo@gmail.com

${ }^{5}$ Orientador, Professor Universidade Federal de Santa Catarina (UFSC). E-mail: alsilva@cca.ufsc.br
} 


\section{INTRODUÇÃO}

Diversas mutações foram encontradas no germoplasma vitícola do Estado de Santa Catarina, como, por exemplo, a Niágara Branca, que difere da Niágara Rosada pela coloração das bagas, a Grano d'Oro, que difere da Bordô pelo vigor e produtividade, a Isabel Precoce, que se diferencia da Isabel pelo menor ciclo, e a uva Itália, com suas diferentes mutações de coloração das bagas, como a Rubi, Benitaka, Red Meire e Brasil (SCHUCK et al., 2009).

A variedade Bordô, originária dos EUA, é uma das principais videiras de Vitis labrusca. $\mathrm{Na}$ década de 1850, despertou interesse dos viticultores europeus devido à resistência ao oídio (Uncinula necator), moléstia que naquela época causava enorme prejuízo à viticultura mundial (GRIGOLETTI; SÔNEGO, 1993).

Sua expansão deu-se devido à fácil adaptação à variabilidade de condições edafoclimáticas, à boa produtividade, longevidade e relativa rusticidade (CAMARGO, 1994). É bastante demandada (32.000 t) para elaboração de vinho tinto, suco, vinagre, geleias e, por sua precocidade, é consumida in natura (RIZZON et al., 2000). Mesmo assim, os trabalhos publicados sobre a uva e o vinho Bordô são restritos (GONÇALVES et al., 1999).

As condições de solo e clima brasileiros favorecem o crescimento vegetativo excessivo das videiras, o que exige dos produtores uma atividade considerável com remoção de folhas e despontes sucessivos. Nesse sentido busca-se reguladores de crescimento que tenham um efeito na redução do vigor das plantas.

A Prohexadiona de cálcio (3-óxido-4-propionil-5-oxo-3-ciclohexeno-carboxilato) é um inibidor da biossíntese de giberelinas, é comercializada pela empresa BASF com o nome de Apogee ${ }^{\circledR}$ e é muito utilizada na cultura da macieira e da pereira, nos Estados Unidos e na Europa, para reduzir o crescimento vegetativo (BYERS; YODER, 1999; OWENS; STOVER, 1999; COSTA et al., 2001). A prohexadiona de cálcio atua na hidroxilação $3-\beta$ da $\mathrm{GA}_{20}$ para $\mathrm{GA}_{1}$. Seu principal efeito é um aumento nos níveis de $\mathrm{GA}_{20}$, que é móvel, mas inativa, e a redução na

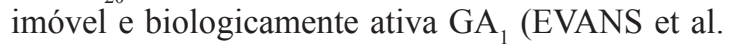
1999).

O objetivo deste experimento foi avaliar a eficiência de diferentes épocas de aplicação da Prohexadiona de Cálcio na redução do vigor e seus efeitos na produtividade e na qualidade da uva da uva Grano d'Oro cultivada em Santa Catarina.

\section{MATERIAL E MÉTODOS}

O experimento foi conduzido em um vinhedo comercial localizado no município de Nova Trento SC (2715’34”'S, 48 $56^{\prime} 54^{\prime \prime} \mathrm{O}$, altitude $\left.78 \mathrm{~m}\right)$, durante a safra de 2010/2011.

O trabalho foi executado com a Grano d'Oro, mutação da variedade Bordô (Vitis labrusca L.). As videiras, conduzidas no sistema de latada, tinham seis anos de plantio e foram plantadas em pé-franco no espaçamento de $3,0 \times 2,0 \mathrm{~m}$. O delineamento utilizado foi de blocos ao acaso, com 4 blocos e 20 plantas por parcela.

Foi utilizado o produto Apogee ${ }^{\circledR}(27,5 \%$ de Prohexadiona de Cálcio) e a concentração de Prohexadiona de Cálcio foi de $250 \mathrm{mg} \mathrm{L}^{-1}$.

Os tratamentos consistiram em:

1 - uma aplicação na plena florada;

2 - uma aplicação 45 dias após a plena florada;

3 - testemunha sem aplicação de prohexadiona de cálcio.

As medidas do comprimento dos ramos iniciaram-se quinze dias após a plena florada. Ramos de 10 plantas por bloco foram marcados antes da aplicação dos tratamentos e medidos quinzenalmente para determinar sua curva de crescimento.

A colheita ocorreu em 25 janeiro de 2011 e, nesse momento cada planta foi colhida individualemente e foi determinado o número de cachos e a produtividade por planta $\left(\mathrm{kg} \mathrm{planta}^{-1}\right)$; em seguida, foi calculada a produtividade estimada por hectare. No momento da colheita, foram amostrados quinze cachos por parcela de forma aleatória para a realização das análises físicas. No laboratório, foi determinado o número de bagas por cacho e, com o uso de balança analítica, determinou-se o peso de cinquenta bagas. No momento da colheita, foram coletadas, 100 bagas por parcela, localizadas na zona basal, mediana e apical de diferentes cachos, tanto do setor leste como do setor oeste das filas, segundo metodologia proposta por Rizzon e Miele (2002).

A partir do mosto das bagas, foram realizadas as seguintes análises em triplicata, conforme metodologia proposta por OIV (1990):

- Acidez total titulável (ATT): para a sua determinação, foi utilizada a metodologia de titulação, em que se adicionaram $5 \mathrm{ml}$ de mosto, $75 \mathrm{ml}$ de água destilada e 2 gotas de fenolftaleína (1\%). Sob agitação, uma solução de hidróxido de sódio $(\mathrm{NaOH}$ $0,1 \mathrm{~N}$ ) foi adicionada até a mudança na coloração.

- Sólidos Solúveis Totais (SST - ${ }^{\circ}$ Brix): através da leitura direta com refratômetro digital de bancada - modelo Instrutherm- RTD -45. O aparelho foi calibrado com água destilada e, em seguida, o mosto 
foi distribuído sobre o prisma, a leitura foi realizada diretamente em ${ }^{\circ}$ Brix.

- $\mathrm{pH}$ : foi avaliado através da leitura das amostras do mosto em pHmetro de bancada - modelo MP 220 Metler-Toledo, calibrado com soluções-tampão a $\mathrm{pH} 4,0$ e $\mathrm{pH} 7,0$.

Todas as análises foram realizadas No laboratório de Morfogênese e Bioquímica Vegetal do Centro de Ciências Agrárias (CCA) da Universidade Federal de Santa Catarina (UFSC).

Os dados obtidos foram submetidos a análise de variância (ANOVA), e ao teste de Tukey, a 5\% de probabilidade de erro para determinar as diferenças entre os tratamentos.

\section{RESULTADOS E DISCUSSÃO}

Foi observada uma redução no crescimento vegetativo de acordo com a época de aplicação da Prohexadiona de Cálcio. A aplicação na plena florada reduziu o crescimento dos ramos em 33\%, aos 15 dias após a plena florada, a redução no crescimento vegetativo diminuiu ao longo do ciclo, sendo que, aos 93 dias após a plena florada,chegou a 8\%, em relação aos outros dois tratamentos (Figura 1).

Os resultados obtidos neste trabalho confirmam o que foi encontrado por Lo Giudice et al. (2003), que observaram uma redução no crescimento dos ramos de videiras da espécie Vitis vinifera; contudo, essa redução no crescimento não eliminou a necessidade da realização do desponte.

Para produtividade (Tabela 1) a aplicação na plena florada $\left(21,9 \mathrm{~kg}_{\text {planta }} \mathrm{e}^{-1}\right.$ e $\left.36,9 \mathrm{~T}^{\mathrm{ha}} \mathrm{h}^{-1}\right)$ foi superior a testemunha $\left(19,2 \mathrm{~kg}_{\text {planta }}{ }^{-1}\right.$ e $\left.31,9 \mathrm{~T} \mathrm{ha}^{-1}\right)$ e a aplicação aos 45 dias após a plena florada $(19,1$ $\mathrm{kg}$ planta $^{-1}$ e 31,8 $\mathrm{T} \mathrm{ha}^{-1}$ ), sendo que as últimas não diferiram entre si.

O número de cachos por planta também foi influenciado pela época de aplicação da Prohexadiona de Cálcio (Tabela 1). As plantas que receberam a aplicação na plena florada apresentaram o maior número de cachos por planta (189) e foram estatisticamente superiores às plantas que receberam aplicação 45 dias após a plena florada e a testemunha (166 e 165 respectivamente). A aplicação na plena florada apresentou 88,8 bagas por cacho e foi estatisticamente superior aos demais tratamentos (Tabela 2).

Os resultados encontrados neste trabalho diferem dos obtidos por Lo Giudice et al. (2004), que observaram que aplicações de Prohexadiona de Cálcio no período da floração podem ter um efeito significativo na redução da produtividade. Contudo, acredita-se que esse aumento na produtividade possa estar relacionado com a redução no vigor das plantas, visto que diversos autores relatam que a produtividade se correlaciona de forma negativa com o vigor da planta (WOLF; POOL, 1988; PAREJO et al., 1995).

Não houve diferenças significativas entre os tratamentos para a variável peso de 50 bagas (Tabela $1)$.

Para a variável sólidos solúveis totais (Tabela 2), foi observado que a aplicação aos 45 dias após a plena florada $\left(14,4{ }^{\circ} \mathrm{Brix}\right)$ apresentou valores estatisticamente superiores à aplicação na plena florada (13,3 ${ }^{\circ}$ Brix), mas não diferiu da testemunha $(13,6$ ${ }^{\circ} \mathrm{Brix}$ ).

Acredita-se que o atraso no acúmulo de açúcar observado no tratamento com aplicação de Prohexadiona de Cálcio na plena florada esteja relacionado com uma situação de vigor reduzido e excessiva carga de frutos (SAMPAIO, 2007). Essa situação foi observada na Austrália, onde, em situações de estresse, plantas pouco vigorosas induziram a um menor acúmulo de açúcar nas bagas quando comparadas com uvas originadas de plantas mais equilibradas e de maior vigor (WITHING, 2004).

Para as variáveis $\mathrm{pH}$ e acidez total titulável, não houve diferenças significativas entre os tratamentos (Tabela 2). E os valores obtidos neste trabalho estão de acordo com os obtidos por Gonçalves et al. (1999), quando estudaram a variedade Bordô. 


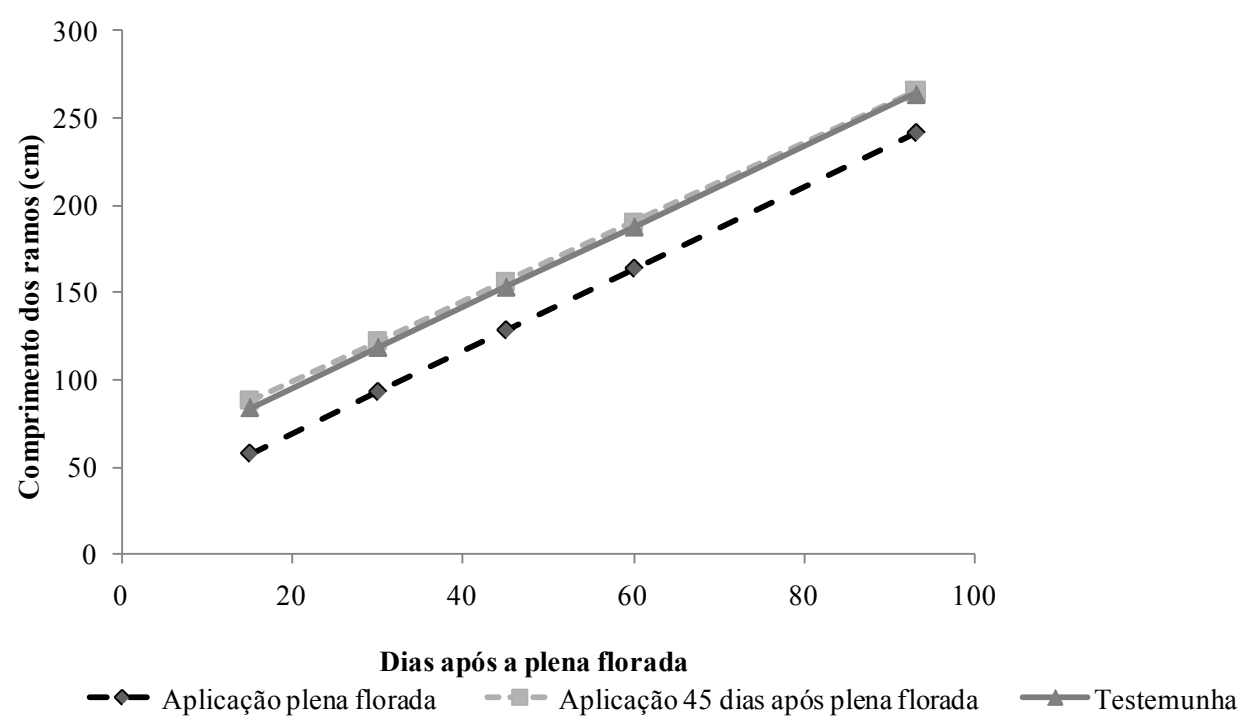

FIGURA 1 - Efeito de diferentes épocas de aplicação da Prohexadiona de Cálcio no crescimento dos ramos das plantas de Grano d'Oro. Florianópolis, 2011.

TABELA 1- Produtividade (kg planta-1 e ton há-1), peso de cachos (g) e peso de 50 bagas (g) de uvas de Grano d'Oro, submetidas a diferentes épocas de aplicação de Prohexadiona de Cálcio, na safra de 2010/2011. Florianópolis, 2011.

\begin{tabular}{lcccc}
\hline Tratamento & $\begin{array}{c}\text { Produtividade } \\
\left(\mathrm{kg} \mathrm{planta}^{-1}\right)\end{array}$ & $\begin{array}{c}\text { Produtividade } \\
\left(\mathrm{T} \mathrm{ha}^{-1}\right)\end{array}$ & $\begin{array}{c}\mathrm{N}^{\circ} \text { Cachos } \\
\text { Planta }^{-1}\end{array}$ & $\begin{array}{c}\text { Peso 50 Bagas } \\
(\mathrm{g})\end{array}$ \\
\hline Aplicação Plena Florada & $21,9 \mathrm{a}$ & $36,5 \mathrm{a}$ & $189 \mathrm{a}$ & $118,0 \mathrm{a}$ \\
Aplicação 45 Dias Após Plena Florada & $19,1 \mathrm{~b}$ & $31,8 \mathrm{~b}$ & $166 \mathrm{~b}$ & $135,1 \mathrm{a}$ \\
Testemunha & $19,2 \mathrm{~b}$ & $32,0 \mathrm{~b}$ & $165 \mathrm{~b}$ & $136,7 \mathrm{a}$ \\
\hline C.V. (\%) & 15,7 & 15,7 & 11,0 & 8,57 \\
\hline
\end{tabular}

* Médias seguidas por letras minúsculas distintas na coluna diferem entre si, pelo Teste de Tukey, a 5\% de probabilidade de erro para o fator tratamento.

TABELA 2 - Número de bagas por cacho, teor de sólidos solúveis ( ${ }^{\circ}$ brix), acidez total (meq $\mathrm{L}^{-1}$ ) e pH de uvas de Grano d'Oro, submetidas a diferentes épocas de aplicação de Prohexadiona de Cálcio, na safra de 2010/2011. Florianópolis, 2011.

\begin{tabular}{lcccc}
\hline Tratamento & $\begin{array}{c}\text { Número de } \\
\text { Bagas } \\
\text { por Cacho }\end{array}$ & $\begin{array}{c}\text { Sólidos Solúveis } \\
\text { Totais } \\
\left({ }^{\circ} \text { Brix }\right)\end{array}$ & $\begin{array}{c}\text { Acidez Total } \\
\text { Titulável } \\
\left(\text { meq L }^{-1}\right)\end{array}$ & $\mathrm{pH}$ \\
\hline Aplicação Plena Florada & $88,8 \mathrm{a}$ & $13,3 \mathrm{~b}$ & $68,0 \mathrm{a}$ & $3,34 \mathrm{a}$ \\
Aplicação 45 Dias Após Plena Florada & $60,0 \mathrm{~b}$ & $14,4 \mathrm{a}$ & $66,0 \mathrm{a}$ & $3,27 \mathrm{a}$ \\
Testemunha & $61,9 \mathrm{~b}$ & $13,6 \mathrm{ab}$ & $62,0 \mathrm{a}$ & $3,27 \mathrm{a}$ \\
\hline C.V. $(\%)$ & 13,8 & 4,1 & 10,17 & 2,14 \\
\hline
\end{tabular}

* Médias seguidas por letras minúsculas distintas na coluna diferem entre si, pelo Teste de Tukey, a 5\% de probabilidade de erro para o fator tratamento. 


\section{CONCLUSÕES}

1. A aplicação da Prohexadiona de Cálcio na plena florada causou as maiores reduções no vigor e o maior aumento na produtividade das plantas de Grano d'Oro.

2. O aumento da produtividade e a redução do vigor foram acompanhados por uma redução nos teores de sólidos solúveis totais.

3. A redução no vigor não eliminou a necessidade da realização do desponte.

\section{REFERÊNCIAS}

BYERS, R. E.; YODER, K. S. Prohexadione-calcium inhibits apple, but not peach, tree growth, but has little influence on apple fruit thinning or quality. HortScience, Alexandria, v.34, p.1205-1209, 1999.

CAMARGO, U.A.. Uvas do Brasil. Brasília: Empresa Brasileira de Pesquisa Agropecuária, Uva e Vinho - SPI, 1994. 90p. (Documentos, 09)

COSTA, G.; ANDREOTTI, C.; BUCCHI, F.; SABATINI, E.; BAZZI, C.; MALAGUTI, S.; RADEMACHER, W. Prohexadione calcium: Growth regulation and reduced fire blight incidence in pear. Hortscience, Alexandria, v.6, p.931-933, 2001.

EVANS, J.R.; EVANS, R. R.; REGUSCI, C. L. Mode of action, metabolism, and uptake of BAS $125 \mathrm{~W}$ prohexadione-calcium. HortScience, Alexandria, v.34, p.1200-1201, 1999.

GONÇALVES, C.A.A.; REGINA, M. de A.; CHALFUN, N.N.J.; ALVARENGA, A.A.; ABRAHÃO, E.; BERZOTI, E. Comportamento da cultivar Folha de Figo (Vitis labrusca L.) sobre diferentes porta-enxertos de videira. Revista Brasileira de Fruticultura, Jaboticabal, v.24, n.4, p.7-14, 1999.

GRIGOLETTI Jr., A.; SÔNEGO, O.R. Principais doenças fúngicas da videira no Brasil. Bento Gonçalves: EMBRAPA CNPUV, 1993. 36p. (Circular Técnica, 17).

LO GIUDICE, D.; WOLF, T. K.; MARINI, R. P. Vegetative response of Vitis vinifera to prohexadione-calcium. HortScience, Alexandria, v.38, p.1435$1438,2003$.
LO GIUDICE, D.; WOLF, T. K.; ZOECKLEIN, B. W. Effects of Prohexadione-calcium on Grape Yield Components and Fruit and Wine Composition. American Journal of Viticulture and Enology, Davis, v.55, p.73-83, 2004.

OIV - Office International de la Vigne et du Vin. Recueil des methodes internatioanles d'analyse des vinset des mouts. Paris, 1990.

OWENS, C. L.; STOVER, E. Vegetative growth and flowering of young apple trees in response to Prohexadione-calcium. HortScience, Alexandria, v.34, p.1194-1196, 1999.

PAREJO, J.; MINGUEZ, S.; SELLA, J.; ESPINAS, E. Sixteen years of monitoring the cultivar Xarello (Vitis vinifera L.) on several rootstocks. Acta Horticulturae, The Hague, v.388, p.123-128. 1995.

RIZZON, L. A.; MIELE, A.; MENEGUZZO, J. Avaliação da uva cv. Bordô para a elaboração de vinho tinto. Ciência e Tecnologia de Alimentos, Campinas, v.20, n.1, p.115-121, 2000.

RIZZON, L. A.; MIELE, A. Avaliação do cv. Cabernet Sauvignon para a elaboração de vinho tinto. Ciência e Tecnologia de Alimentos, Campinas, v.22, n.2, p.192-198, 2002.

SAMPAIO, T. L. B. Using rootstocks to manipulate vine physiological performance and mediate changes in fruit and wine composition. 2007. $240 \mathrm{f}$. Tese (PHD) - Oregon State University, Oregon, 2007.

SCHUCK, M.R.; MOREIRA, F.M.; GUERRA, M.P.; VOLTOLINI, J.A.; GRANDO, M.S.; LIMA DA SILVA, A. Molecular characterization of grapevine from Santa Catarina State using microsatellite markers. Pesquisa Agropecuária Brasileira, Brasília, v. 44, p. 487-495, 2009.

WHITING, J. R. Grapevine rootstocks. In: DRY, P.R.; COOMBE, B.G. (Ed.). Viticulture: resources in Australia. Ashford: Winetitles, 2004. v.1, p. 167-188.

WOLF, T. K.; POOL, R. M. Effects of rootstock and nitrogen fertilization on the growth and yield of Chardonnay grapevines in New York. American Journal of Enology and Viticulture, Davis, 39: 29-37. 1988. 